\title{
A Bandwidth Statistical Multiplexing and Control Method for Satellite Broadcasting
}

\author{
Yingqiang Wang $\left(\mathbb{D},{ }^{1}\right.$ Zhaohua Nian, ${ }^{1}$ Chang Liu, ${ }^{1}$ Wei Han, ${ }^{1}$ and Maowei Lin ${ }^{2}$ \\ ${ }^{1}$ Space Star Technology Co., Ltd., Beijing, China \\ ${ }^{2}$ China Meteorological Administration, Beijing, China \\ Correspondence should be addressed to Yingqiang Wang; wangyq@spacestar.com.cn
}

Received 8 April 2020; Revised 14 June 2020; Accepted 7 July 2020; Published 1 August 2020

Academic Editor: Chenxi Huang

Copyright (c) 2020 Yingqiang Wang et al. This is an open access article distributed under the Creative Commons Attribution License, which permits unrestricted use, distribution, and reproduction in any medium, provided the original work is properly cited.

\begin{abstract}
In the field of satellite data broadcasting, the management quality of data broadcasting bandwidth is directly related to the throughput of the broadcasting system and plays an important role in the performance of satellites. In this paper, for the sunsynchronous orbit meteorological satellite broadcasting which has the conventional product files and emergency information, a broadcast bandwidth statistical multiplexing and control method is designed for bandwidth management. It can be used for the management of broadcasting between regular products and emergency information, as well as internal broadcasting among regular products. This paper is the first to apply common multiplexing of PID and channel mode (CMPCM) to satellite broadcasting. The test verified that the broadcast channel of the parameters and the broadcast schedule management channel resources achieved statistical multiplexing of bandwidth, ratio of channel management functions, and data broadcast control. Broadcasting occupation ratio (BOR) and broadcasting file error ratio (BER) improved significantly. This is significant for improving the efficiency of satellite uplink broadcasting.
\end{abstract}

\section{Introduction}

The weather is playing an increasingly important role whether in people's daily life or in the operation of the entire national economy. Meteorological disasters cause huge losses to people's lives, property, and national economic development every year.

Now with the development of China's meteorological service, meteorological disaster monitoring, and early warning process, the satellite data broadcasting system as a whole is an important subsystem in the system; it is not only an important domestic and foreign meteorological data transmission distribution system, but also the ground platform for the distribution of observational data, radar data, satellite data, and other data, as well as the broadcasting and transmission of regular meteorological products and emergency information. The satellite data broadcasting system can provide FENGYUN series of meteorological satellite observation data and product broadcasts for different levels of meteorological departments and users in the Asia-Pacific region. The data and products are formatted, packaged, modulated, upconverted, and amplified in real time. The satellite data broadcasting system operates automatically under the bidirectional driving of data and mission schedules and has a highly reliable, all-weather service operation capability.

Broadcast bandwidth is an important scarce resource for satellites especially for geostationary satellite. The total bandwidth of geostationary satellite transponder's link is very limited [1]. Compared with the speed of sun-synchronous orbit sensing satellites, which is often hundreds of megabytes or even several gigabytes, its rates are usually only several megabytes to tens of megabytes. For continuous broadcasting, even $7 * 24$ hours, broadcast method becomes very important. Therefore, whether the data broadcasting system can reasonably utilize the satellite bandwidth becomes particularly important. The level of data broadcasting efficiency is the key to the system's ability to complete the 
daily broadcasting task. How to use these tens of megabytes of data bandwidth efficiently is an important content in the development of satellite data broadcasting systems and plays a key role in the application of meteorology.

In order to cope with the signal delay of the satellite link, Donner et al. [2] proposed and analyzed a suitable solution based on the use of a performance enhancing proxy (PEP) and header compression for voice over IP (VoIP) streams to save satellite bandwidth. Park and Kim proposed an adaptive bandwidth allocation scheme according to traffic demands and weather-induced signal attenuation, which can achieve a reasonable solution between the maximization of total capacity and the support of proportional fairness among the beams for satellite downlinks [3]. In [4], Lei and VazquezCastro proposed an analytical solution for the optimal carrier allocation and improved it in terms of power gain, spectral efficiency, and traffic matching ratio compared with the conventional system, which is designed based on uniform bandwidth and power allocation. The issue of multibeam power allocation based on traffic demands and channel conditions over satellite downlinks with power and delay constraints is addressed [5]. Li et al. [6] investigated an energy harvesting scheme in a smart grid based on the cognitive relay protocol, developing the analytically optimal bandwidth allocation strategy to maximize the total sum rate of the grid network. Bahnasse et al.'s article contains information on a new intelligent bandwidth allocation model for future network (Smart Allocation) [7]. The included data describe the topology of the network testbed and the obtained results.

Shi et al. proposed two joint power and bandwidth allocation schemes [8] based on the intercluster orthogonal frequency reuse, which considers channel conditions and the traffic demands of user beams. It gives a good compromise between efficiency of frequency bands utilization and cochannel interference mitigation. The joint bandwidth and power allocation iterative algorithm based on duality theory was proposed [9] to obtain the optimal solution of satellite power and bandwidth resources in a multi-spot-beam satellite communication system. A joint power and bandwidth allocation with QoS support algorithm was proposed by Miao [10] using convex optimization method for heterogeneous wireless networks. It satisfied the minimum rate constraint of delay-constraint service traffic and proportional fairness of best-effort service traffic. With the help of the classical multiknapsack problem, heuristic algorithms together with some innovative scaling schemes [11] are presented for the optimization of resource allocation in systems using DVB-S2 links.

These conclusions cannot be directly extended to the meteorological satellite broadcasting, especially for data broadcasting. In satellite broadcasting systems, logical channel is used to manage different types of broadcast data. The types of information in different broadcast channels have different requirements on broadcast timeliness, which can spread minutes to hours. At the same time, the allocation of bandwidth needs to change dynamically with time and be adjusted in real time. The bandwidth of the channel which has no unbroadcast data needs to release resources in time for reuse by other channels.

\section{Demand Introduction}

Bandwidth reuse statistics are one of the most important functions in a data broadcasting system. Bandwidth reuse statistics function has an important influence on whether the precious satellite channel resources can be fully utilized [12], whether the weather data can reach the destination for the first time, and whether data is correctly transmitted. The system must transmit meteorological regular files and emergency information at the same time and ensure that the bandwidth of the satellite is fully utilized as far as possible. Control of content delivery needs to be prioritized, to ensure that urgent tasks can be issued as soon as possible. The total rate of satellite data broadcasting should reach or approach the maximum bandwidth of the satellite channel as much as possible and make full use of the satellite channel resources, so that all types of meteorological data can reach the receiving station in the shortest time.

Based on the concepts of channel maximum bandwidth, minimum bandwidth, and channel priority, CMACast broadcasting system designed and implemented the bandwidth allocation strategy mode which changes with time [13, 14], which has guiding significance for this paper.

2.1. Functional Requirements. The data that the satellite broadcasting system needs to distribute belongs to multiple different PIDs and channels, which are at different data levels and are subject to different priorities and bandwidth restrictions. To achieve high-performance broadcasting, the system must use an optimized bandwidth statistics algorithm and adjust the broadcast speed of each data packet in real time to make it reach or approach the theoretical broadcast upper limit.

File channels involved in this article include 5 categories: HRIT-1 (high rate information transport 1), HRIT-2, HRIT3, LRIT (low rate information transport), and EWAIB (emergency weather alert information broadcast). Among them, HRIT-1 and HRIT-3 include regular files and emergency information, HRIT-2 contains regular files only, and EWAIB contains emergency information [15]. Table 1 shows detailed information.

Data broadcasting system software completes the configuration of the parameters of the channel, scheduling management, and broadcasting channel resources and realizes the bandwidth statistical multiplexing function, the channel ratio management function, and the data broadcasting control function. The specific functions are as follows:

(1) Bandwidth Statistical Multiplexing. The bandwidth statistical multiplexing function can adjust the broadcast rate of each channel in real time according to the set bandwidth statistical multiplexing strategy and the priority of each channel, as well as the highest and lowest bandwidth parameters during the data broadcast process. It can also allocate 
TABLe 1: Channel and types.

\begin{tabular}{lcc}
\hline Channel & Content & Type \\
\hline \multirow{3}{*}{ HRIT-1 } & LMI level 2 products & Emergency \\
& AGRI level 1 products & Regular \\
& HRIT-1 timetable & Regular \\
& Satellite information & Regular \\
GIIRS level 2 products & Regular \\
HRIT-2 & AGRI level 2 products & Regular \\
& HRIT-2 timetable & Regular \\
& Satellite information & Regular \\
& AGRI level 1 products & Regular \\
HRIT-3 & LMI level 2 products & Emergency \\
& HRIT-3 timetable & Regular \\
& Satellite information & Regular \\
LRIT & AGRI LRIT data & Regular \\
& Satellite information & Regular \\
EWAIB & LRIT timetable & Regular \\
& Emergency information & Emergency \\
\hline
\end{tabular}

conventional broadcast channels and emergency information broadcast. The transmission rate between channels guarantees the rational use of satellite channel bandwidth.

(2) Channel Ratio Management. Channel ratio management function can be calculated according to the statistical multiplexing of bandwidth theoretical rate channel for each file and the respective channel number data for the mixing process.

(3) Data Broadcast Control. Data broadcast control function may control the broadcast code rate for each broadcast's threads, to ensure that thread data code rate can be broadcasted as expected.

Bandwidth statistical multiplexing includes statistical multiplexing among regular file channels and statistical multiplexing between regular files and emergency information. The statistical multiplexing among the regular file channels mainly calculates the theoretical rate of each channel in real time according to the priority of the file channel, the highest (lowest) bandwidth setting, and the dynamic changes of the channel data. The broadcast of regular files gives the bandwidth needed for emergency information broadcast through its internal statistical reuse by reducing the total code rate of regular file broadcast. When the emergency information broadcast completes, the regular file broadcast rate is automatically restored.

The function of bandwidth multiplexing statistics is calculating the real-time broadcast occupied bandwidth of each channel and reallocating the remaining bandwidth according to the priority of each channel. While achieving dynamic balance, the total bandwidth will occupy the satellite channel bandwidth as much as possible, so that the entire data broadcasting performance is optimal.

Bandwidth statistical multiplexing and speed control are a feedback control process, in which regular data and emergency data adjust real-time broadcasting speed due to available bandwidth, as shown in Figure 1.

Emergency information and regular file channel information are combined for bandwidth multiplexing and

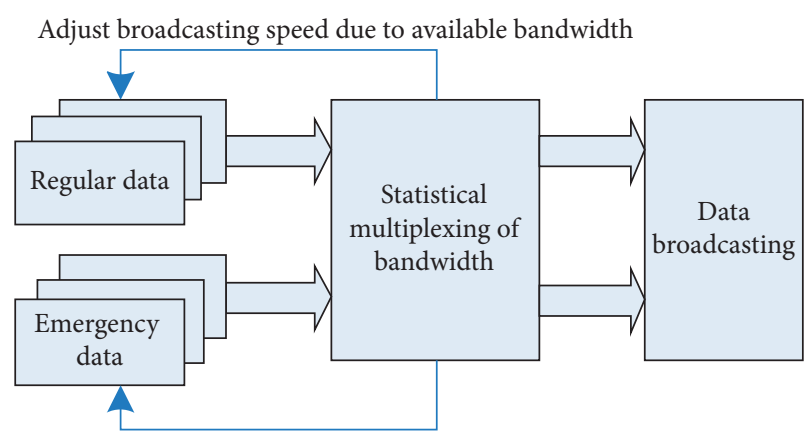

FIgURE 1: Bandwidth statistical multiplexing process control.

control, which requires priority to ensure that emergency information channels have sufficient bandwidth, and then the remaining bandwidth is allocated among each regular file channel.

2.2. Nonfunctional Requirements. For bandwidth statistics and multiplexing control method, the broadcasting system needs to achieve the following indicators:

(1) Speed of broadcast for emergency messages and general files is $12 \mathrm{Mbps}$ for HRIT-1, $10 \mathrm{Mbps}$ for HRIT-2, 2 Mbps for HRIT-3, $256 \mathrm{Kbps}$ for LRIT, and $2 \mathrm{Kbps}$ for EWAIB.

(2) The utilization rate of bandwidth is more than $96 \%$. In an independent link, the bandwidth sum of different PIDs and channels should not be less than 96\% of the total bandwidth.

(3) Meanwhile, file security also needs to be satisfied, and broadcasting success rate should be $100 \%$. Each broadcasting file must be completely processed, and the data received through the small station are exactly the same as the data before broadcasting.

\section{System Design}

The data broadcasting system provides the principle of statistical multiplexing and priority setting among different broadcasting channels for satellite data broadcasting, which can easily set the channel rate and priority. When there is channel access and exit, the speed of other channels can be automatically adjusted according to the current bandwidth usage and the priority of different channels to achieve the full use of satellite channel bandwidth resources.

For the design of bandwidth statistical multiplexing, factors that need to be considered include the following:

(1) The Use of Satellite Channel Bandwidth Should Be Maximized. In the process of statistical multiplexing, the first thing considered is that the total rate of satellite data broadcasting should reach or approach the maximum rate allowed by the satellite channel as much as possible, make full use of the satellite 
channel, and be able to broadcast meteorological products at the fastest speed.

(2) During the Process of Bandwidth Statistical Multiplexing, the Total Out Bandwidth is Stable and Free from Fluctuations. During statistical multiplexing, it is necessary to ensure that the overall rate of satellite data broadcasting is stable and that the instantaneous total code rate will not increase or decrease due to the rate adjustment of regular files or emergency information, resulting in data loss or waste of bandwidth.

(3) Bandwidth Statistics Multiplex Response Is Fast and Time-Effective. The purpose of bandwidth multiplexing statistics is to adjust the code rate of related channels when the channel that broadcasts regular files or emergency information changes, to ensure continuous and stable broadcasting of data on each channel. Therefore, bandwidth multiplexing statistics need to ensure high timeliness and respond quickly to changes in the status of each channel.

(4) Bandwidth Statistical Multiplexing Does not Affect the Accuracy of the Data. In the process of bandwidth statistical multiplexing, changing the code rate of each channel will not cause data broadcast errors or data loss due to improper code rate control.

With a full understanding of the requirements and principles of bandwidth statistical multiplexing, this paper has designed a variety of algorithms for bandwidth statistical multiplexing mechanisms, which can make all regular files or emergency information channels use the same bandwidth statistical multiplexing mechanism. Based on a unified priority, each file channel and each emergency information channel are allocated bandwidth according to different mechanisms.

A channel is a logical division of data. When data are finally sent, different channels data are combined into one channel and sent out in the form of network data packets. Bandwidth (code rate) of each channel is expressed as a statistical average rate over a period of time. In implementation, the rate setting of the channel adopts network packets to match the proportion of the code rate of the channel for the entire bandwidth. The specific algorithm is as follows:

(i) Let the rate of $N$ channels be $\mathrm{BR}_{1}-\mathrm{BR}_{n}$ and the total rate be $\mathrm{BR}=\sum \mathrm{BR}_{i}$, the proportion of each channel in the total bandwidth is

$$
b_{i}=\frac{\mathrm{BR}_{i}}{\mathrm{BR}}
$$

(ii) It has $m$ network packets as a working period.

(iii) In each period, every channel participates based on the whole package.

(iv) Channel $i$ sends $P_{\mathrm{i}}$ packets in a matching period:

$$
P_{i}=\left[M \times b_{i}+0.5\right] \text {. }
$$

The flow of bandwidth statistical multiplexing and channel bandwidth control is shown in Figure 2.

\section{Internal Bandwidth Multiplexing among Regular Files}

In this paper, according to the characteristics of regular files, without considering PID, the bandwidth statistical multiplexing among channels is mainly designed as follows.

4.1. Absolute Priority. The principle of the absolute priority algorithm is that when bandwidth multiplexing is performed, allocation is completely based on priority. When bandwidth is allocated, the channel with the highest priority is first allocated to the maximum bandwidth, and the remaining bandwidth is allocated to the next priority.

4.2. Priority Weight Allocation Mechanism. The priority weight allocation mechanism is an improvement strategy of the absolute priority mechanism. The absolute priority mechanism is only related to the level of priority, and the specific value of the priority has no practical significance for the mechanism. In the priority weight allocation mechanism, each priority has a weight, which indicates the proportion of bandwidth allocated under the priority. When performing actual multiplexing statistics, the value of the priority is quantified as a weight. Channel weights are allocated for each channel bandwidth.

\subsection{Bandwidth Allocation Set by Channel Mechanism.} The bandwidth allocation algorithm set according to the channel is similar to the prior weight allocation mechanism. The difference is that the weight of the bandwidth allocation is determined by the set channel bandwidth. The bandwidth allocation is based on the priority from high to low. Channel weights are allocated for each channel bandwidth.

Obviously, the characteristics of the three mechanisms are as follows:

(i) Based on the absolute priority mechanism, a channel with a higher priority can obtain high bandwidth, and a channel with a lower priority can obtain additional bandwidth only when all other channels have obtained the highest bandwidth.

(ii) Based on the priority weight allocation mechanism, the remaining bandwidth can be allocated to each channel in proportion. Even for channels with low priority, additional bandwidth can be obtained by setting the weight.

(iii) Based on the bandwidth ratio allocation set by channels mechanism, the actual rate of each channel approaches its maximum bandwidth when the bandwidth is relatively sufficient.

In summary, when PID is not considered, each mechanism has its own advantages. When the system is running, different priority mechanisms can be selected to meet the 


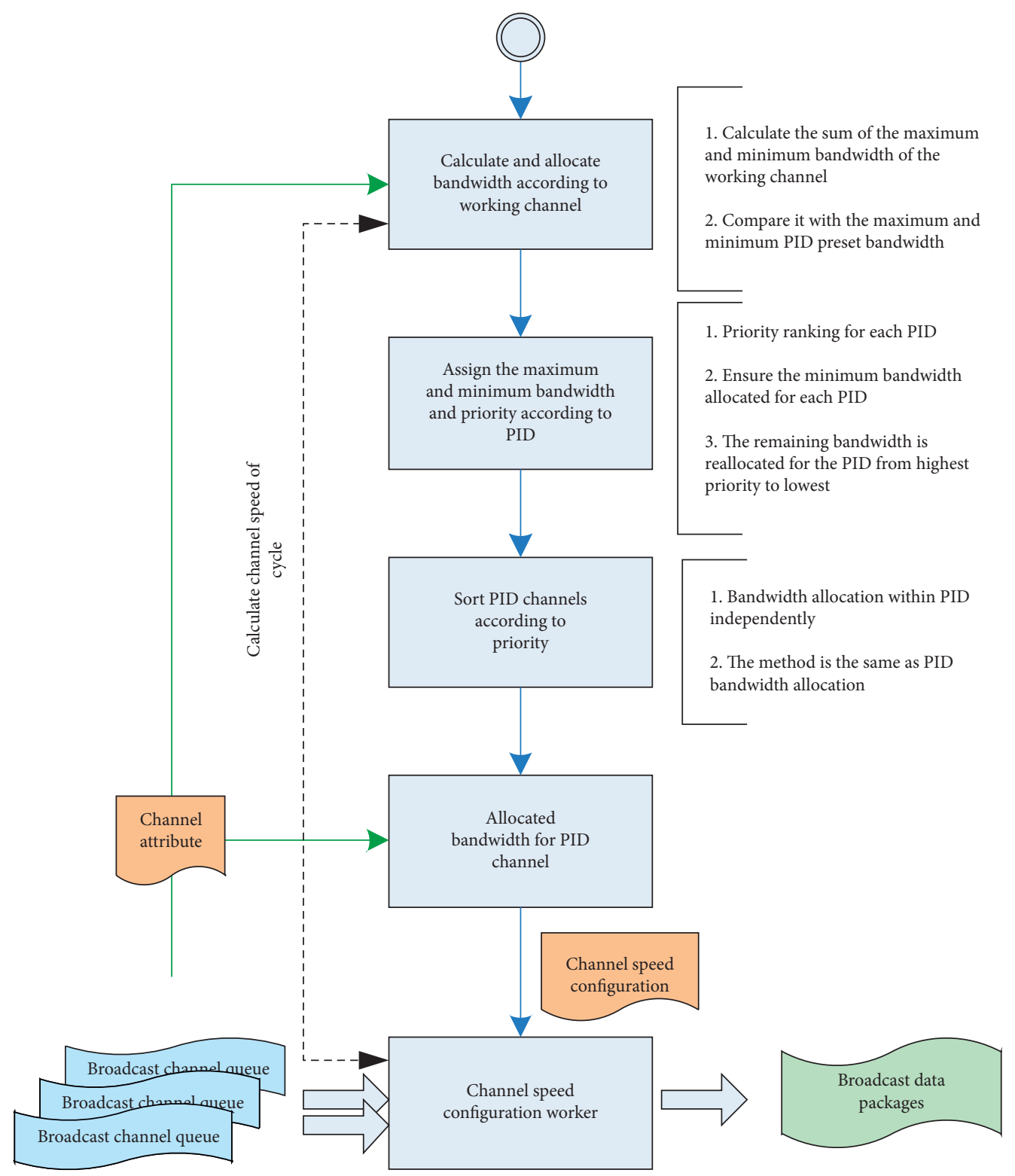

FIgURE 2: Bandwidth and statistical multiplexing channel control process.

needs of data broadcast. At the same time, it may customize the priority mechanism differently from the above strategy.

After considering the system PID, channel statistical multiplexing has two-level statistical multiplexing, i.e., PID and channel. In order to adapt to different broadcast requirements, this scheme designs two kinds of bandwidth statistical multiplexing modes between PID and channel, which are described as follows.

4.4. PID First and Then Channel Mode (PFM). PID first and then channel refers to first calculating the theoretical rate of each PID according to the bandwidth range, priority, and statistical multiplexing strategy set by each PID and then calculating the theoretical rate of all channels under the PID according to the theoretical bandwidth of each PID. In this mode, the channels between PIDs do not directly participate in multiplexing statistics and can control the rate of each PID better.

4.5. Common Multiplexing of PID and Channel Mode (CMPCM). This mode does not calculate the PID rate separately but instead substitutes the priority of the PID into the channel below it for global multiplexing statistics. This method enables higher priority channels to obtain higher rates, without being constrained by the PID bandwidth range.

The following is experimental tests for the two bandwidth statistical multiplexing methods designed. The configuration information of the participating devices is shown in Table 2. 
TABLE 2: Bandwidth multiplexing test channel configurations.

\begin{tabular}{lcc}
\hline Name & Model & Configuration \\
\hline Broadcast server & Inspur NF5280M4 & CPU: E5-8860*4 \\
& & Memory: 16 GB \\
OS: SUSE x64 \\
$\begin{array}{l}\text { Data modulator } \\
\text { Demodulator }\end{array}$ & Newtec M6100 & \\
Newtec AZ910 & CPU: Xeon E5-2620 *2 \\
Receiver & HP Z820 & Memory: 4 GB \\
& & OS: Windows 7 \\
\hline
\end{tabular}

Table 3: The PID bandwidth and priorities.

\begin{tabular}{lccc}
\hline PID & Minimum bandwidth & Maximum bandwidth & Priority \\
\hline PID1 & 26 & 64 & 1 \\
PID2 & 26 & 64 & 2 \\
\hline
\end{tabular}

In the experiment, two PIDs were designed, with two channels under each PID, and a total bandwidth of $64 \mathrm{Mbps}$ was set. The specific configurations of the PID and channels are shown in Tables 3 and 4.

The test results of the actual broadcast rate of each PID and channel after statistical bandwidth multiplexing in different ways are shown in Figure 3, and the statistical multiplexing algorithm uses an absolute priority mechanism.

From the occupation of the total bandwidth of the four channels, the following conclusions can be drawn:

(i) First PID after passage way for PID theoretical rate was well controlled at the PID level to ensure PID bandwidth, so that each PID having high priority within the channel gets extra bandwidth.

(ii) PID priority is taken into account in CMPCM. PID priority is higher than channel priority. In this experiment, the order is $A>B>C>D$.

\section{Bandwidth Multiplexing between Regular Files an Emergency Information}

In order to ensure the timely broadcasting of emergency information, the bandwidth of emergency information must be guaranteed first for the statistical multiplexing between regular files and emergency information. According to this principle, this paper designs the following three statistical multiplexing algorithms between regular files and emergency information.

5.1. No Delay Mechanism (NDM). In order to guarantee that receiving station can receive and process emergency information broadcasted by the master station, master station broadcasts information instantly without delay and buffering.

However, because the code rate of emergency information may not be obtained in advance, in order to ensure that the maximum broadcast bandwidth does not exceed the channel bandwidth capacity, when the master station starts to receive and forward emergency information, it
TABle 4: The PID channel bandwidth and priorities.

\begin{tabular}{llccc}
\hline PID & $\begin{array}{c}\text { Channel } \\
\text { number }\end{array}$ & $\begin{array}{c}\text { Minimum } \\
\text { bandwidth } \\
(\mathrm{Mbps})\end{array}$ & $\begin{array}{c}\text { Maximum } \\
\text { bandwidth } \\
\text { (Mbps) }\end{array}$ & Priority \\
\hline \multirow{2}{*}{ PID1 } & Channel A & 8 & 32 & 1 \\
& Channel B & 8 & 32 & 2 \\
PID2 & Channel C & 8 & 32 & 1 \\
& Channel D & 8 & 32 & 2 \\
\hline
\end{tabular}

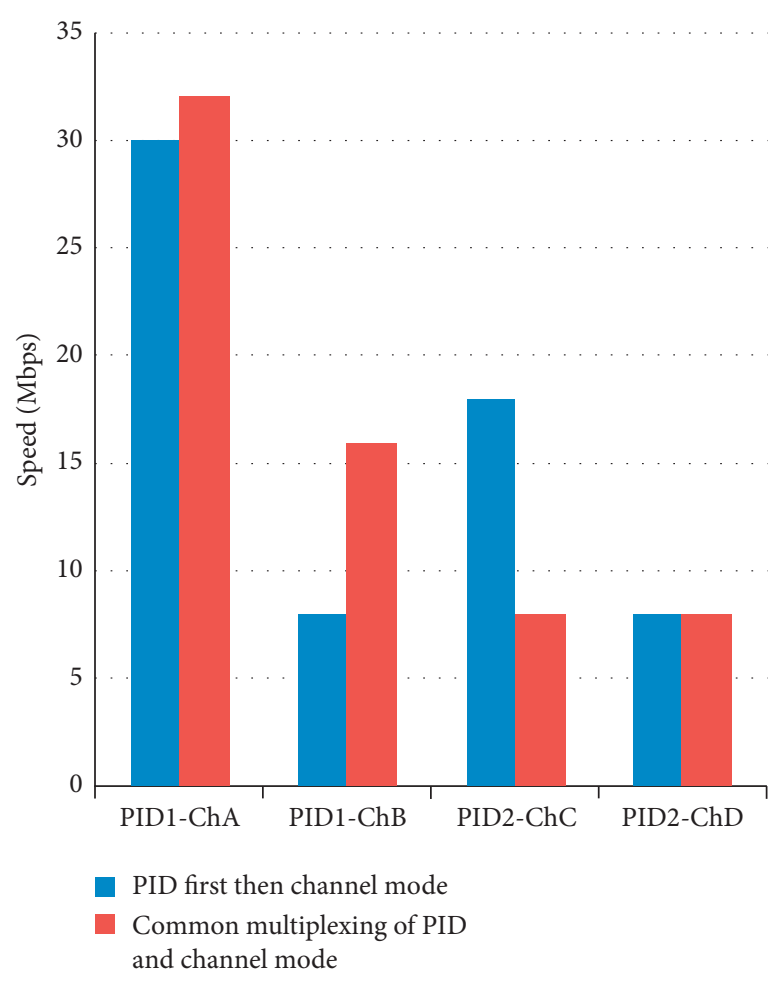

Figure 3: Two bandwidth occupation modes.

needs to allocate the emergency information bandwidth according to the set maximum bandwidth of the emergency information channel. After the master station software calculates the emergency information, it needs to release the emergency information highest bandwidth and the difference between the emergency information and the actual bandwidth.

In this way, there is no time delay from the master station receiving the emergency information data and it can almost start broadcasting, but this may result in a short period of channel bandwidth waste due to calculating the emergency information data symbol rate. If it is receiving data from the modulator encoder, the data broadcasting system software can obtain the modulator setting rate in advance and adjust the maximum broadcast bandwidth accordingly, which can effectively avoid bandwidth waste.

5.2. No Bandwidth Wasting Mechanism (NBWM). No channel bandwidth wasting mechanism is different from the processing flow at the beginning. When receiving emergency information, the master station broadcast is not performed, 
but rather the data is buffered until the actual code rate is calculated. The bandwidth is allocated according to the actual rate of the emergency information, and broadcasting starts from the beginning of the buffer tail.

This mechanism will not cause the waste of channel bandwidth, but it will have a delay when the receiving end stations obtain emergency information. The delay time equals the calculation time of the emergency information code rate.

5.3. No Delay and No Waste Mechanism (NDNM). No delay and no waste mechanism is similar to NBWM. When the master station starts receiving the emergency information, there is no broadcasting or buffering; only emergency information actual code rate is calculated. According to the actual rate, bandwidth is allocated and then the most recently received data is directly broadcasted. The previously received data are no longer broadcasted and discarded directly.

This mechanism can achieve no delay and no waste of bandwidth, but it will lose some data at the beginning of the emergency information data flow.

In summary, the three types of bandwidth statistical multiplexing mechanisms between regular files and emergency information have their own characteristics, and the corresponding mechanism needs to be selected according to the actual situation, which can make good use of the channel bandwidth and reduce the delay.

In general, the broadcasting without delay mechanism is suitable for emergency information flow. Most of this data needs live broadcasting, and the shorter the delay, the better. No channel bandwidth wasting mechanism is suitable for broadcasting in advance to make good the emergency information file; emergency information file before the broadcast may not get an accurate broadcasted rate, it is not easy to avoid the waste of bandwidth through the bandwidth settings, and such data requires less time delay, so it is reasonable to use no channel bandwidth wasting mechanism. No delay and no bandwidth waste mechanism is suitable when the emergency information beginning has unimportant data, such as redundant data and so on.

Emergency information in this paper contains lightning data and earthquake, flood, mudslide, and other meteorological disaster prediction information. With the requirements of small amount of data and low latency, the broadcasting without delay mechanism is used for the broadcasting.

Since the satellite data cycle is one hour, one hour test time is taken. The specific test results are shown in Figure 4.

Figure 4 shows that NBWM has some time delays, NDNM discards data beginning. All these situations are unacceptable during broadcasting. Therefore, we conclude that NDM is better than the other mechanisms.

\section{Experiment}

6.1. Environment Introduction. In order to verify the broadcasting strategy designed in this paper, we use

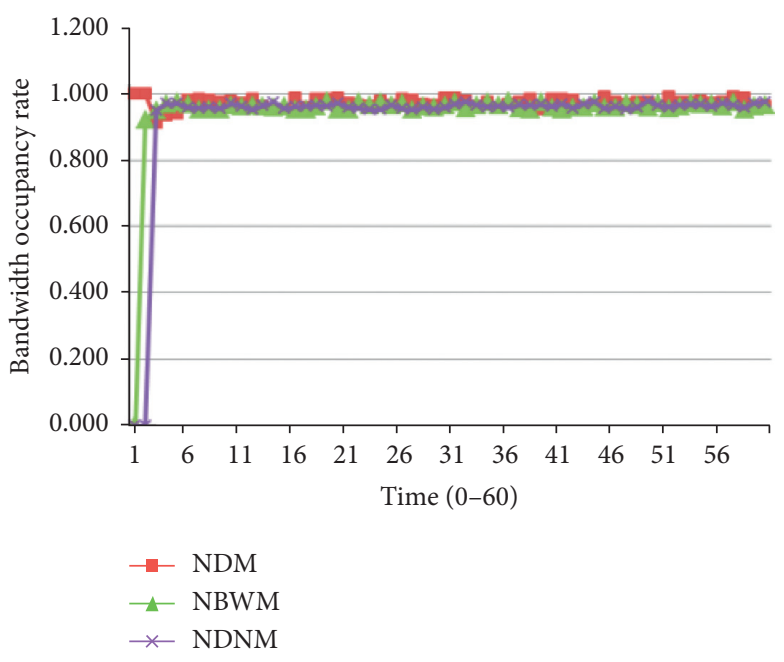

FIgURE 4: Test results of the three mechanisms.

common multiplexing of PID and channel mode among regular files, and broadcasting without delay mechanism between regular files and emergency information. Data broadcasting software and receiving software were developed, and FENGYUN 4-A satellite was used in this experiment.

We transmitted the broadcast data to the satellite repeater through DVB-S2 modulator, frequency upconverter, power amplifier equipment, and a 13 meter antenna and finally received the data through a frequency downconverter and a DVB-S2 demodulator using a small antenna, as shown in Figure 5.

Since the design principles and mechanisms of HRIT1, HRIT-2, HRIT-3, LRIT, and EWAIB have no difference, the test part of this paper takes HRIT-1 as a typical object, and its test results can represent the test conditions of other file channels. The performance configuration of the equipment participating in the test is consistent with Table 2.

The broadcast speed of HRIT- 1 is $12 \mathrm{Mbps}$; then one hour data is about $42 \mathrm{~Gb}$. Experiment takes 378 files with 5637144576 total bytes. This paper adopts four combinations of experiment for HRIT-1.

The following four test scenarios are designed: Test 1 contains a single-PID file broadcasted with $12 \mathrm{Mbp}$. Test 2 contains regular files broadcasted with 4 PIDs. Each rate is $3 \mathrm{Mbps}$ and the total rate is $12 \mathrm{Mbps}$. Test 3 contains regular files broadcasted with different code rate PIDs: $1 \mathrm{Mbps}$, $2 \mathrm{Mbps}$, $4 \mathrm{Mbps}$, and $5 \mathrm{Mbps}$. Test 4 contains regular files broadcasted with 3 PIDs, each rate is $3.3 \mathrm{Mbps}, 1 \mathrm{PID}$ is used for emergency information broadcasting with the rate $0.1 \mathrm{Mbps}$, and the total rate is $10 \mathrm{Mbps}$.

6.2. Metrics for Evaluation. During the test, the following indicators are used to evaluate and compare the bandwidth statistics and multiplexing results.

Broadcasting occupation ratio (BOR) is defined as the ratio between the total bandwidth allocated by the channel and the maximum bandwidth allowed by the network: 


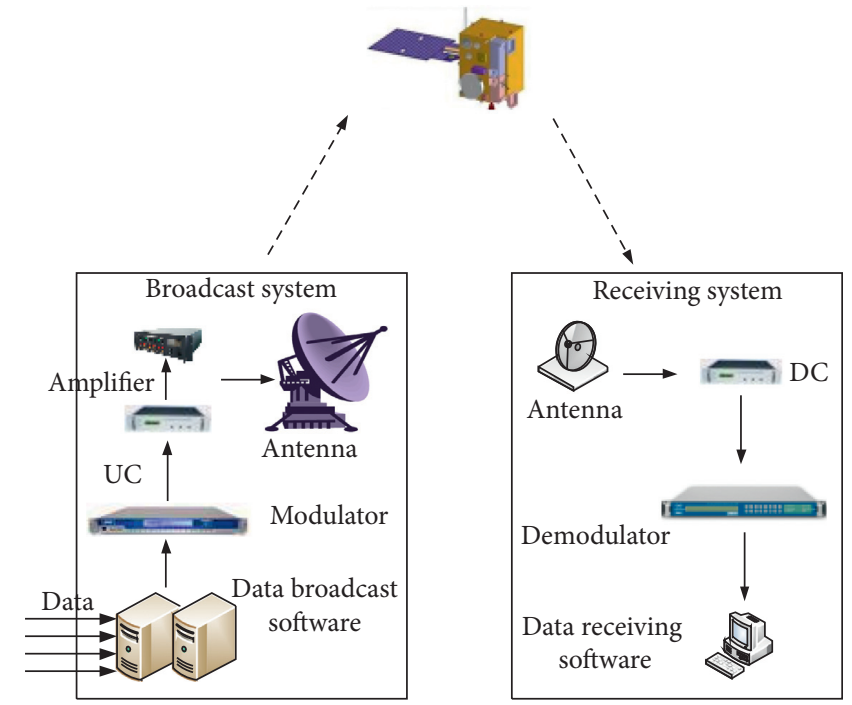

FIgURE 5: Satellite broadcast test block diagram.

TABLE 5: The experiment results.

\begin{tabular}{|c|c|c|c|c|c|c|c|c|c|}
\hline Test no. & PID & $\mathrm{BF}_{i}$ & $\mathrm{BS}_{i}$ (byte) & $\mathrm{BR}_{i}$ (Mbps) & $\mathrm{BO}_{i}(\mathrm{Mbps})$ & $\mathrm{BOR}_{i}$ & $\mathrm{BFR}_{i}$ & $\mathrm{BSR}_{i}$ & $\mathrm{BER}_{i}$ \\
\hline \multirow[t]{2}{*}{1} & PID1 & 378 & 5637144576 & 12 & 11.652 & 0.971 & 1 & 1 & 0 \\
\hline & PID1 & 92 & 1409185367 & 3 & 2.923 & 0.974 & 1 & 1 & 0 \\
\hline \multirow{4}{*}{2} & PID2 & 95 & 1408351758 & 3 & 2.915 & 0.972 & 1 & 1 & 0 \\
\hline & PID3 & 93 & 1412392349 & 3 & 2.932 & 0.977 & 1 & 1 & 0 \\
\hline & PID4 & 98 & 1407215102 & 3 & 2.92 & 0.973 & 1 & 1 & 0 \\
\hline & PID1 & 30 & 469785314 & 1 & 0.974 & 0.974 & 1 & 1 & 0 \\
\hline \multirow{4}{*}{3} & PID2 & 63 & 933895146 & 2 & 1.938 & 0.969 & 1 & 1 & 0 \\
\hline & PID3 & 119 & 1823684108 & 4 & 3.885 & 0.971 & 1 & 1 & 0 \\
\hline & PID4 & 166 & 2409780008 & 5 & 4.865 & 0.973 & 1 & 1 & 0 \\
\hline & PID1 & 115 & 1859703744 & 3.9 & 2.949 & 0.983 & 1 & 1 & 0 \\
\hline \multirow{3}{*}{4} & PID2 & 119 & 1862197390 & 4 & 2.933 & 0.978 & 1 & 1 & 0 \\
\hline & PID3 & 112 & 1842063726 & 3.9 & 2.945 & 0.982 & 1 & 1 & 0 \\
\hline & PID4 & 32 & 73179716 & 0.2 & 0.969 & 0.969 & 1 & 1 & 0 \\
\hline
\end{tabular}

Broadcasting file ratio (BFR) is defined as the ratio of the number of files received by the small station to the total number of files broadcasted:

$$
\mathrm{BFR}=\sum_{i} \frac{\mathrm{BF}_{i}}{\mathrm{BF}} .
$$

Broadcasting file size ratio (BSR) is defined as the ratio of the total number of file bytes received by the small station to the total number of bytes broadcasted:

$$
\mathrm{BSR}=\sum_{i} \frac{\mathrm{BS}_{i}}{\mathrm{BS}} .
$$

Broadcasting file error ratio (BER) is defined as the ratio of the sum of error bytes in the received file of the small station to the total number of bytes broadcasted:
6.3. Experiment Results. The meteorological products for HRIT-1, HRIT-2, HRIT-3, and LRIT cycle once an hour, so all the tests last for one hour, and the results are shown in Table 5.

According to Table 5, some figures can be drawn from these numbers, such as Figures 6-8.

From the data shown above, it can be concluded that the total broadcast rate is very stable, there is no obvious fluctuation, and BOR is also very accurate, nearly $97 \%$.

6.4. Performance Evaluation. It can be concluded from Table 5 that BFR is 1 , BSR is 1 , and BER is 0 , which proves that the regular files and emergency information can be completely and safely broadcasted to the channel equipment and the satellite. 


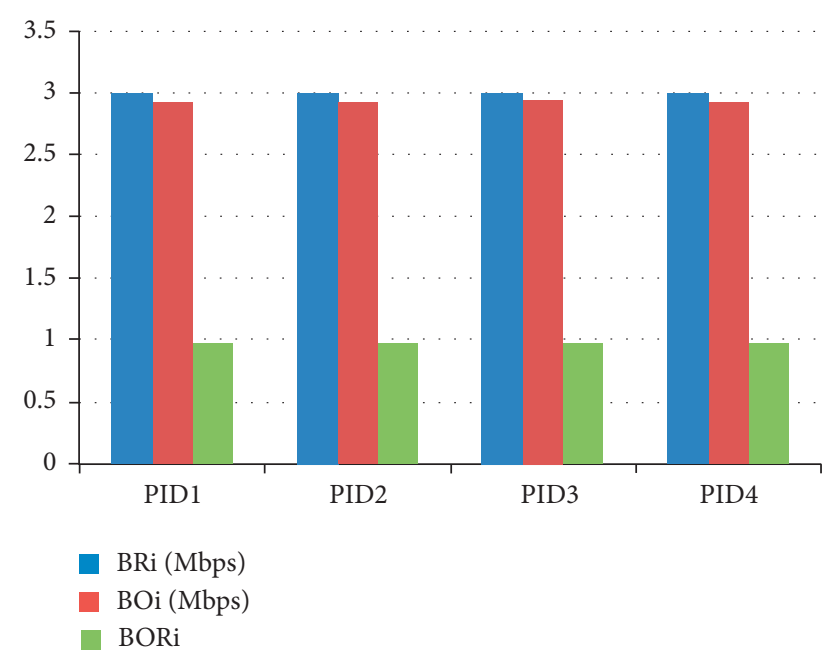

Figure 6: Average bandwidth tests.

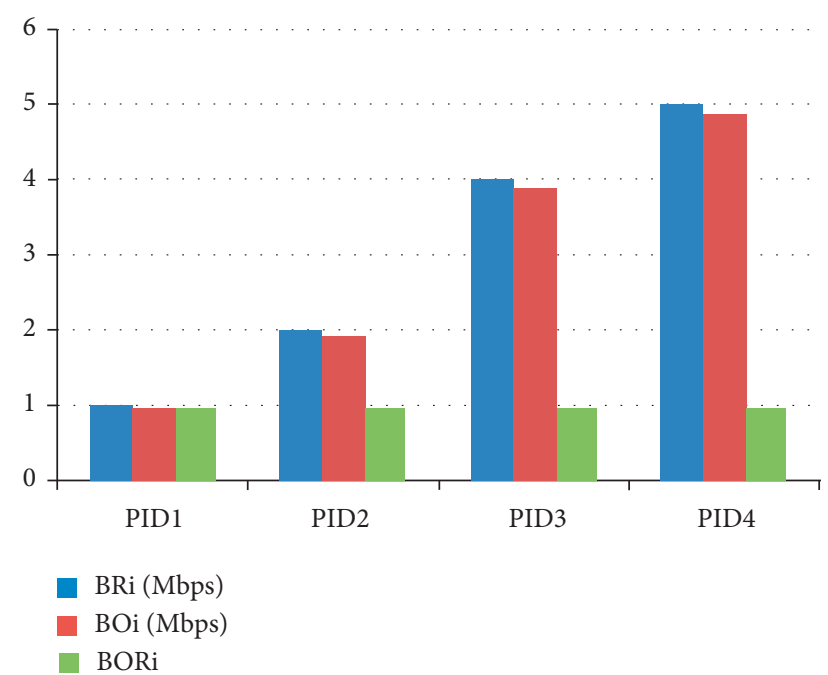

Figure 7: Staircase bandwidth tests.

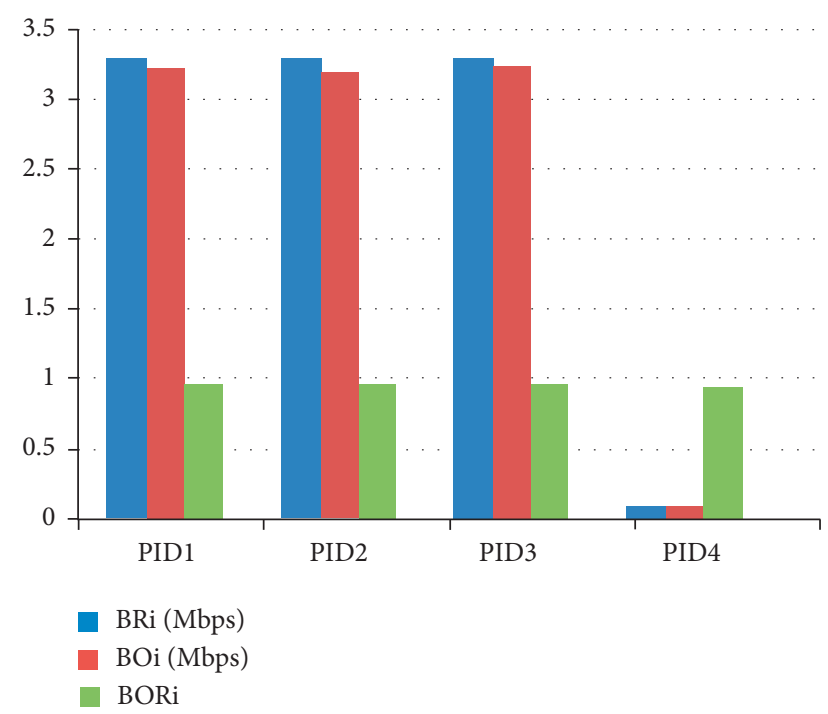

Figure 8: Regular files and emergency information tests.
Figures 6-8 indicate that during the four-combination test, whether single-PID or multi-PID combination, the bandwidth occupation rate of each channel is about $97 \%$, which is better than the requirement utilization rate with 96\% described in Section 2.2.

Accordingly, this bandwidth statistics and multiplexing control method meets the broadcasting system nonfunctional requirements well.

\section{Conclusion}

Satellite bandwidth multiplexing statistics are one of the most important functions of satellite data broadcasting platform. The statistical function of bandwidth multiplexing has an important impact on whether the satellite channel resources can be fully utilized, whether the meteorological data can arrive at the destination for the first time, and whether data is correctly transmitted.

This paper introduced a broadcast bandwidth statistical multiplexing and control method. Common multiplexing of PID and channel mechanism is used for regular file data broadcasting. Broadcasting without delay mechanism is used for emergency information broadcasting. However, broadcast timetable, track data, user authorization, and other information are not taken into account; they do not belong to regular or emergency information.

This broadcast bandwidth management improves satellite broadcasting efficiency. It has become an effective transmission means of meteorological information broadcasting service. It also has meaningful reference for the sunsynchronous orbit satellite communication.

Our future work will include analyzing the characteristics of all broadcast data, considering uplink and downlink quality monitoring [4], and adding adaptive adjustment function for transmission power by the aid of knapsack allocation problem [8], for the purpose of realizing the optimal allocation of broadcast resources.

\section{Data Availability}

The data used to support the findings of this study are included within the article.

\section{Conflicts of Interest}

The authors declare that there are no conflicts of interest regarding the publication of this paper.

\section{Acknowledgments}

The authors wish to acknowledge the cooperation of China Meteorological Administration National Satellite Meteorological Center. This work was supported in part by the National Natural Science Foundation of China Youth Fund under Grants 51608209 and 18BTJ031.

\section{References}

[1] D. Roddy, Satellite Communications, McGraw-Hill, New York, NY, USA, 2001. 
[2] A. Donner, J. A. Saleemi, and J. Mulero Chaves, "TETRA backhauling via satellite: improving call setup times and saving bandwidth," Journal of Computer Networks and Communications, vol. 2014, Article ID 562546, 16 pages, 2014.

[3] U. Park, H. W. Kim, D. Sub Oh, and B. J. Ku, "A dynamic bandwidth allocation scheme for a multi-spot-beam satellite system," ETRI Journal, vol. 34, no. 4, pp. 613-616, 2012.

[4] J. Lei and M. Vazquez-Castro, "Joint power and carrier allocation for the multibeam satellite downlink with individual SINR constraints," in Proceedings of the 2010 IEEE international conference on communications (ICC), Cape Town, South Africa, May 2010.

[5] J. P. Choi and V. W. S. Chan, "Optimum power and beam allocation based on traffic demands and channel conditions over satellite downlinks," IEEE Transactions on Wireless Communications, vol. 4, no. 6, pp. 2983-2993, 2005.

[6] Y. Li, Z. Wang, L. Luo, Z. Chen, B. Xia, and H. Luo, "Bandwidth allocation of cognitive relay networks with energy harvesting for smart grid," Journal of Computer Networks and Communications, vol. 2019, Article ID 5038963, 9 pages, 2019.

[7] A. Bahnasse, F. E. Louhab, H. A. Oulahyane, M. Talea, and A. Bakali, "Smart bandwidth allocation for next generation networks adopting software-defined network approach," Data in Brief, vol. 20, pp. 840-845, 2018.

[8] S. Shi, G. Li, Z. Li, H. Zhu, and B. Gao, "Joint power and bandwidth allocation for beam-hopping user downlinks in smart gateway multibeam satellite systems," International Journal of Distributed Sensor Networks, vol. 13, no. 5, Article ID 155014771770946, 2017.

[9] H. Wang, A. Liu, and X. Pan, "Optimization of joint power and bandwidth allocation in multi-spot-beam satellite communication systems," Mathematical Problems in Engineering, vol. 2014, Article ID 683604, 9 pages, 2014.

[10] J. Miao, Z. Hu, K. Yang, C. Wang, and H. Tian, "Joint power and bandwidth allocation algorithm with Qos support in heterogeneous wireless networks," IEEE Communications Letters, vol. 16, no. 4, pp. 479-481, 2012.

[11] S. I. Wayer and A. Reichman, "Resource management in satellite communication systems: heuristic schemes and algorithms," Journal of Electrical and Computer Engineering, vol. 2012, Article ID 169026, 10 pages, 2012.

[12] L. Peng and J. Ye, "A bandwidth allocation scheme for the integrated service satellite broadcast network," Microcomputer Information, vol. 24, no. 15, pp. P10-P16, 2008.

[13] ran Liu, K. Jiang, and Y. Hu, "Design of data broadcast platform in China meteoroligical administration's satellite data broadcast system(CMACast)," Digital Communication World, vol. 7, no. 7, pp. P54-P58, 2013.

[14] C. Wang, Li Xiang, Y. Chen, and K. Jiang, "Design of CMA's broadcast system for meteorological data-CMACast," Journal of Applied Meteorological Science, vol. 23, no. 1, pp. P113P120, 2012.

[15] Z. Xu, Di Xian, Y. Gao, and Y. Qi, "Design and implementation of FENGYUN-4 satellite data service system," Satellite Application, vol. 83, no. 11, pp. P55-P60, 2018. 